\title{
Translation, revision, and validation of the Chinese version of the Satisfaction with Oral Anti-Diabetic Agent Scale (C-SOADAS) in patients with type 2 diabetes mellitus
}

This article was published in the following Dove Press journal:

Patient Preference and Adherence

Yi-Ju Lin'

Chin-Yuan Wang ${ }^{2}$

Elizabeth H Chang ${ }^{1,3,4}$

Ssu-Wei Cheng ${ }^{5}$

Yu Kol,4

'Department of Clinical Pharmacy, School of Pharmacy, College of Pharmacy, Taipei Medical University, Taipei, Taiwan; ${ }^{2}$ Department of Internal Medicine, Division of Endocrinology and Metabolism, National Taiwan University Hospital, Taipei, Taiwan; ${ }^{3}$ Department of Pharmacy, Wan Fang Hospital, Taipei Medical University, Taipei, Taiwan; ${ }^{4}$ Research Center for Pharmacoeconomics, College of Pharmacy, Taipei Medical University, Taipei, Taiwan; ${ }^{5}$ Department of Pharmacy, Shin Kong Wu Ho-Su Memorial Hospital, Taipei, Taiwan
Correspondence: Yu Ko

Department of Clinical Pharmacy, School of Pharmacy, College of Pharmacy, Taipei Medical University, 250 Wu-Hsing Street, Taipei II 031 , Taiwan

Tel +8862273 6I66I ext 6I74

Email nancyko@tmu.edu.tw
Objective: The aim of this study was to translate, adapt, and validate the Satisfaction with Oral Anti-Diabetic Agent Scale (SOADAS) in type 2 diabetes mellitus (DM) patients taking oral antidiabetic drugs (OADs) in Taiwan.

Patients and methods: The SOADAS was translated to Chinese and was modified based on reviews of two physicians, five diabetes educators, and two patient focus groups. A crosssectional interviewer-administered survey was conducted in adult patients with type $2 \mathrm{DM}$ who were taking OADs. The Chinese version of the SOADAS (C-SOADAS), the EuroQol 5 dimensions 3-level (EQ-5D-3L) questionnaire, and a demographic questionnaire were administered to participants. Instrument structure, internal consistency, convergent validity, and known-group validity were assessed.

Results: A total of $260 \mathrm{DM}$ patients were recruited. The mean score of an individual item ranged from 3.6 to 3.9, while the mean total score (out of 25 possible points) was 18.7 points. Overall, floor and ceiling effects were negligible. The Cronbach's $\alpha$ value was 0.81 . All the four predetermined hypotheses for known-group validity assessment were fulfilled. In convergent validity testing, the C-SOADAS total scores were found to be correlated with EuroQol-Visual Analog Scale (EQ-VAS) scores $(r=0.2 ; p<0.01)$ but not with EuroQol 5 dimensions (EQ-5D) index scores $(r=0.02 ; p=0.81)$.

Conclusion: The 5-item C-SOADAS appears to be a psychometrically acceptable measure of OAD treatment satisfaction among type $2 \mathrm{DM}$ patients in Taiwan. The tool may be incorporated into clinical practice to quickly assess treatment outcomes from patients' perspectives.

Keywords: diabetes mellitus, oral antidiabetic agent, satisfaction, validation, reliability, C-SOADAS, Taiwan

\section{Introduction}

Diabetes mellitus (DM) is a chronic disease that causes the body to deteriorate overtime. More than 400 million people live with DM worldwide. ${ }^{1}$ The global DM prevalence has increased from $4.7 \%$ in 1980 to $8.5 \%$ in 2014 , and it is estimated that the significant impact of DM will affect approximately 180 million people by $2025^{2,3}$ A similar increase has been observed in Taiwan with the DM prevalence rising from $4.8 \%$ in $2007,6.4 \%$ in 2009 , and $7.1 \%$ in 2012 to $10.0 \%$ in $2015 .{ }^{1,4}$

Given that patients' perceptions of treatment outcomes may vary from clinicians' judgments, ${ }^{5}$ it has become an important approach to evaluate treatment outcomes from patients' perspectives using instruments to assess patients' satisfaction or health-related quality of life (HRQoL). In addition, compared to generic measures, disease-specific 
instruments are generally more responsive to changes and can target the concerns of patients with that particular disease. Several patient-reported diabetes-specific instruments have been developed and validated to evaluate the overall impact of DM and its treatment on various aspects of an individual's HRQoL. For example, the audit of diabetes-dependent quality of life is a 19-item HRQoL instrument designed to measure patients' perceptions of DM impact, and this instrument has been translated into more than 20 languages. ${ }^{6}$ Another recommended DM-specific HRQoL measure is the diabetes care profile, which has demonstrated good reliability and validity in a number of studies. ${ }^{7}$

In addition to HRQoL, another important patient-reported outcome is treatment satisfaction, particularly satisfaction with medications. ${ }^{8}$ According to the Centers for Disease Control and Prevention (CDC), patients taking pills only, without insulin, accounted for more than $50 \%$ of DM patients in the US in $2011 .{ }^{9}$ In Taiwan, it is reported that a majority (87.5\%) of DM outpatients take oral antidiabetic drugs (OADs) only. ${ }^{10}$ In spite of the significant and still growing number of patients taking OADs for their DM treatment, few patient-reported DM instruments have targeted OADs either alone or along with the assessment of insulin satisfaction. An exception is the instrument developed and validated by Donatti et al: ${ }^{11}$ the Satisfaction with Oral Anti-Diabetic Agent Scale (SOADAS), which was the first treatment satisfaction instrument specific to OADs. The SOADAS was drafted by focus groups and cognitive debriefing interviews with type 2 DM (T2DM) patients taking OADs, and its psychometric properties were further evaluated in a cross-sectional sample of 106 DM patients and their physicians at their clinics. ${ }^{11}$

In view of escalating DM prevalence and the large proportion of DM patients taking OADs in Taiwan as well as the growing number of OADs available and their increasing uses in clinical practice, we conducted this study with the aim of translating, adapting, and validating the SOADAS in T2DM patients taking OADs in Taiwan.

\section{Patients and methods}

This study is a cross-sectional interviewer-administered survey that was approved by the institutional review board of Shin-Kong Memorial Hospital (approval no SKH20150712R).

\section{Study setting and participants}

The interviews were conducted in the endocrinology and metabolism clinics of an 862-bed medical center in Taipei from October 2016 to April 2017. Patients were recruited if they met the following criteria: 1) diagnosis of T2DM, 2) age 20 years or older, 3 ) taking one or more oral medications for diabetes, and 4) normal cognitive function (ie, no cognitive impairment such as Alzheimer's disease, Parkinson's disease, or dementia). Patients who used solely insulin for DM treatment were excluded.

\section{Survey instrument}

\section{Translation and adaptation of the SOADAS}

The SOADAS is the first treatment satisfaction measure developed specifically for OADs. ${ }^{11}$ The measure contains six items that focus on tolerability, medication's ability to control blood sugar and diabetic symptoms, onset of action, effect on weight, and overall satisfaction. Each item is scored on a 5-point scale ranging from 1 (extremely dissatisfied) to 5 (extremely satisfied). The SOADAS has shown good internal consistency (Cronbach's $\alpha=0.86$ ). In addition, the SOADAS has demonstrated convergent validity, as it was highly correlated with the Treatment Satisfaction Questionnaire for Medication $(r=0.71, p<0.0001)$.

The translation of the SOADAS to Chinese was performed by a professional bilingual translator and revised by a group of health care professionals and two patient focus groups. The health care professionals included two physicians and five diabetes educators with an average of 19.1 practice-years in diabetes care. These experts were interviewed individually and were asked to examine the items for their importance and clarity as well as their relevance and applicability to local DM population. After the interviews, the SOADAS items were revised according to comments received and issues observed. The modifications included the removal of two items and the addition of a new item. Specifically, the health care professionals suggested the elimination of the question about "how quickly the medication controlled blood sugar?" Their reasoning was that patients on OADs do not monitor their blood sugar as regularly as those taking insulin and that DM patients in Taiwan tend to focus more on a drug's effect rather than its onset of action. In addition, the experts suggested removing the question regarding the medication's ability to control diabetic symptoms. The diabetes educators interviewed particularly pointed out that this question was misleading given that patients in Taiwan are educated to control their blood sugar instead of diabetic symptoms. Finally, based on the experts' clinical experience, they advised the addition of a new item that assessed the convenience of taking the OAD, which they believed could influence patients' satisfaction with the drug. The revised SOADAS was subsequently 
tested in two patient focus groups that consisted of a total of seven T2DM patients. The patients confirmed that the revised questions were comprehensible and adequately reflected the characteristics of OADs that they cared about.

The final Chinese version of the SOADAS (C-SOADAS) contained five items that assessed patients' satisfaction with their oral antidiabetic medication's ability to control blood sugar, its effect on weight, the tolerability of its side effects, dosing regimen convenience, and overall satisfaction. Similar to the original SOADAS, each item had response choices presented on a 5-point scale ranging from 1 (extremely dissatisfied) to 5 (extremely satisfied). The scores of the five items were summed to generate a total score ranging from 5 to 25 , with higher scores indicating greater satisfaction.

\section{EuroQol 5 dimensions 3-level (EQ-5D-3L) questionnaire}

The EQ-5D-3L questionnaire is a generic health status measure developed by the EuroQol group which consists of a descriptive system and EuroQol-Visual Analog Scale (EQ-VAS). The descriptive system comprises five dimensions: mobility, self-care, usual activities, pain/discomfort, and anxiety/depression. ${ }^{12}$ Each of the dimensions consists of three levels of response: no problems, some problems, and extreme problems. Responses to the five questions can be used to generate an index score ranging from 0 (death) to 1 (perfect health), where a score of $<0$ represents a health state considered worse than death. The Japan value set (or tariffs) ${ }^{13}$ was applied to compute the EuroQol 5 dimensions (EQ-5D) index score, because Taiwanese weights were not available at the time of study. The EQ-VAS portion of the EQ-5D-3L records respondents' self-rated health on a scale from 1 to 100 , with higher scores indicating a better general health status.

\section{Demographic and clinical characteristics}

In addition to the C-SOADAS and EQ-5D-3L, demographic and clinical data were also collected from the participants and, if available, their medical records, which included age, gender, education, height, weight, monthly income, DM duration (in years), presence of DM-related complications, most recent $\mathrm{HbA1c}(\%)$, adherence to $\mathrm{OAD}(\mathrm{s})$, and whether they had a DM-associated hospitalization or emergency room (ER) visit in the past year. Adherence to OAD therapy was assessed by the following question: "In the past month, have you taken your oral antidiabetic medicine exactly as your doctor told you in terms of timing, dosing, and frequency?" with the response options of "never", "seldom", "sometimes", "often", and "always".

\section{Survey administration}

After enrolling in this study, the participants were informed verbally about the study context and signed written informed consent. Then, they were asked to complete the SOADAS, the EQ-5D-3L, and the questionnaire collecting information about their demographic and clinical characteristics. Upon completion, participants received compensation equivalent to approximately US\$15.

\section{Statistical analyses}

Descriptive statistics were used to examine the respondents' and C-SOADAS items' characteristics. Percentages of respondents who scored the lowest (5 points) and highest (25 points) possible C-SOADAS total scores were calculated to examine floor and ceiling effects. Internal consistency was assessed by Cronbach's $\alpha$ with an $\alpha$-value of $>0.7$ to demonstrate acceptable reliability. ${ }^{14}$

Face validity was confirmed by the health care professionals and patient focus groups at the development phase. Principal component analysis was used to test the unidimensionality of the C-SOADAS. Construct validity was examined by exploratory factor analysis to identify the structure of the C-SOADAS. Convergent validity of the C-SOADAS was evaluated against the EQ-5D index and VAS scores by Pearson correlation. Known-group validity was assessed by examining whether the C-SOADAS could discriminate subgroups with different characteristics. Specifically, we hypothesized that a higher mean C-SOADAS total score would be observed in patients who were in good control of $\mathrm{HbAlc}$ (ie, most recent $\mathrm{HbA} 1 \mathrm{c} \leq 7 \%$ ), had no DMassociated hospitalization/ER visit in the past year, or had fewer DM-related complications. Independent $t$-test and one-way analysis of variance (ANOVA), where appropriate, were used for testing these hypotheses. In addition, Spearman correlation analysis was performed to test the hypothesis that patients' SOADAS total scores were positively associated with their adherence to OADs.

All statistical analyses were performed using IBM SPSS Statistics for Windows, version 19 (IBM Corporation, Armonk, NY, USA).

\section{Results}

\section{Characteristics of study participants}

A total of 260 DM patients participated in the C-SOADAS validation study. A summary of the participants' demographic and clinical characteristics is presented in Table 1 . The mean $( \pm \mathrm{SD})$ age of the participants was $58.0( \pm 11.4)$ years. The proportion of participants who were males $(56.2 \%)$ was 
Table I Characteristics of study participants $(n=260)$

\begin{tabular}{|c|c|c|}
\hline Characteristics & Mean & SD \\
\hline Age (years) & 58.0 & 11.4 \\
\hline DM duration (years) & 9.9 & 7.4 \\
\hline \multirow[t]{2}{*}{$\mathrm{HbAlc}(\%)$} & 8.0 & 1.6 \\
\hline & $\mathbf{n}$ & $\%$ \\
\hline \multicolumn{3}{|l|}{ Gender } \\
\hline Male & 146 & 56.2 \\
\hline Female & 114 & 43.8 \\
\hline \multicolumn{3}{|l|}{ Highest education level } \\
\hline No formal education & 2 & 0.8 \\
\hline Elementary school & 25 & 9.6 \\
\hline Junior high school & 37 & 14.2 \\
\hline Senior high school & 115 & 44.2 \\
\hline Vocational school & 16 & 6.2 \\
\hline College & 50 & 19.2 \\
\hline Master degree & 14 & 5.4 \\
\hline Doctoral degree & I & 0.4 \\
\hline \multicolumn{3}{|l|}{ Monthly income (NTD) } \\
\hline No income & 118 & 45.4 \\
\hline 20,000 (below) & 4 & 1.5 \\
\hline $20,00 I-30,000$ & 16 & 6.2 \\
\hline $30,001-40,000$ & 24 & 9.2 \\
\hline $40,001-50,000$ & 28 & 10.8 \\
\hline $50,00 \mathrm{I}-75,000$ & 25 & 9.6 \\
\hline 75,000 and above & 29 & 11.2 \\
\hline Refuse to report & 16 & 6.2 \\
\hline \multicolumn{3}{|l|}{ DM-related complications } \\
\hline Cardiovascular disease & 93 & 35.8 \\
\hline Diabetic neuropathy & 47 & 18.1 \\
\hline Diabetic retinopathy & 33 & 12.7 \\
\hline Peripheral vascular disease & 25 & 9.6 \\
\hline Kidney disease & 20 & 7.7 \\
\hline Oral complications & 18 & 6.9 \\
\hline \multicolumn{3}{|l|}{ BMI groups } \\
\hline Underweight & 2 & 0.8 \\
\hline Normal & 75 & 28.8 \\
\hline Overweight & 75 & 28.8 \\
\hline Obese & 108 & 41.5 \\
\hline \multicolumn{3}{|l|}{ Number of OADs } \\
\hline 1 & 108 & 44.3 \\
\hline 2 or more & 136 & 55.7 \\
\hline
\end{tabular}

Abbreviations: $\mathrm{BMI}$, body mass index; DM, diabetes mellitus; OADs, oral antidiabetic drugs; NTD, New Taiwan Dollar. slightly larger than that of females $(43.8 \%)$. The mean $( \pm \mathrm{SD})$ DM duration was $9.9( \pm 7.4)$ years. Most of the participants' highest education level was senior high school (44.2\%), followed by college (19.2\%) and junior high school (14.2\%). Approximately half of the participants were obese $(41.5 \%)$, had no income $(45.4 \%)$, and were taking more than one OAD (55.7\%). The most common DM-related complication reported was cardiovascular disease (35.8\%), followed by diabetic neuropathy (18.1\%) and diabetic retinopathy (12.7\%). Approximately two-thirds (65.8\%) of the participants had uncontrolled HbA1c $(>7 \%)$, and the overall mean $( \pm \mathrm{SD}) \mathrm{HbA} 1 \mathrm{c}$ was $8.0 \%( \pm 1.6)$.

\section{Item level and total scores of the C-SOADAS}

Table 2 represents the item level and total scores of the C-SOADAS. The mean score of an individual item ranged from 3.6 to 3.9 , while the mean total score was 18.7 points. Very few participants provided a response of "extremely dissatisfied" to any item, whereas $5.4 \%-13.1 \%$ of study participants responded "extremely satisfied" to individual items. Overall, floor and ceiling effects were negligible, with $0 \%$ and $2.3 \%$ of the patients scoring the lowest and highest possible total scores, respectively. All five items had an itemtotal correlation coefficient of 0.7 or higher.

\section{Reliability and validity assessment}

The Cronbach's $\alpha$ value was 0.81 for the C-SOADAS scores, indicating good internal consistency. Principal component analysis showed that the C-SOADAS was unidimensional with the first factor accounting for $59.3 \%$ of the total variance and an eigenvalue of 3.0, which was significantly higher than that of the second component (eigenvalue $=0.7$ ) and those of the subsequent components. In convergent validity testing, the C-SOADAS total scores were found to be

Table 2 Characteristics of the C-SOADAS $(n=260)$

\begin{tabular}{|c|c|c|c|c|c|c|}
\hline Item number & Mean & SD & $\begin{array}{l}\text { Observed } \\
\text { score }\end{array}$ & Floor (\%) & Ceiling (\%) & $\begin{array}{l}\text { Item total } \\
\text { correlation }(r)^{*}\end{array}$ \\
\hline QI: ability to control blood sugar & 3.6 & 0.9 & $\mathrm{I}-5$ & 1.2 & 13.1 & 0.8 \\
\hline Q2: effect on weight & 3.7 & 0.7 & $2-5$ & 0 & 5.4 & 0.7 \\
\hline Q3: tolerability of the side effects & 3.7 & 0.8 & $\mathrm{I}-5$ & 1.5 & 9.6 & 0.8 \\
\hline Q4: convenience of drug taking & 3.9 & 0.6 & $\mathrm{I}-5$ & 0.4 & 10.4 & 0.7 \\
\hline Q5: overall satisfaction & 3.8 & 0.6 & $2-5$ & 0 & 8.8 & 0.9 \\
\hline Total C-SOADAS score & 18.7 & 2.8 & $10-25$ & 0 & 2.3 & \\
\hline
\end{tabular}

Note: *All $p<0.000$.

Abbreviations: C-SOADAS, Chinese version of the Satisfaction with Oral Anti-Diabetic Agent Scale; Q, question. 
correlated with EQ-5D VAS scores $(r=0.2 ; p<0.01)$ but not with EQ-5D index scores $(r=0.02 ; p=0.81)$.

All the four hypotheses were fulfilled. A higher C-SOADAS total score was associated with good control of HbA1c $(p<0.0001)$, the absence of DM-associated hospitalization/ER visit in the past year $(p=0.049)$, and better adherence to OAD therapy $\left(r_{\mathrm{s}}=0.17 ; p=0.006\right)$. Moreover, the ANOVA result showed that there were significant differences among patients with different numbers of DM-related complications ( $p=0.002)$, and the post hoc Scheffe's test showed that the mean SOADAS total score of the patients with no complication was higher than the scores of those with three or more complications $(p=0.006)$.

\section{Discussion}

This study aimed to translate and revise the original SOADAS to develop a Chinese version where the psychometric properties were then validated in T2DM patients in Taiwan. The study results indicate that $\mathrm{C}$-SOADAS is a reliable measure for the assessment of patient satisfaction with oral antidiabetic medications as indicated by its high internal consistency. In addition, the C-SOADAS demonstrated convergent validity, as shown by its correlation with EQ-5D VAS scores, and known-group validity, given its ability to discriminate among known groups based on adherence, complications, DM control, and ER/inpatient visits.

The C-SOADAS shows psychometric properties similar to those of the original SOADAS developed and validated in the US. ${ }^{11}$ Specifically, both measures had a Cronbach's $\alpha$ of 0.8 , and the factor analysis yielded one factor solution in both studies, with the first factor accounting for approximately $60 \%$ of the total variance. Moreover, ceiling and floor effects were not evident in the C-SOADAS or in the original version, which indicates good discriminative ability. Furthermore, similar to the findings of previous satisfaction studies, ${ }^{15,16}$ higher HbA1c levels were found to be associated with lower treatment satisfaction in the current study. Finally, in the convergent validity assessment, it was found that C-SOADAS total scores were correlated with the EQ-5D VAS scores, but not with EQ-5D index scores. An explanation could be that a large proportion (63.5\%) of our study participants scored the highest possible EQ-5D index score of 1.0, resulting in little variance among the scores and making them less discriminating than the EQ-5D VAS scores.

A notable change to the original SOADAS in the C-SOADAS was the removal of the question about a medication's ability to control diabetic symptoms. Common symptoms of DM include frequent urination, thirst, extreme fatigue, and blurry vision. ${ }^{17}$ Given the fact that more than two-fifths of T2DM patients in Taiwan were more than 65 years of age, ${ }^{18}$ they were likely to perceive these DM symptoms to be part of aging, rather than indicators of OADs' effectiveness. Another difference in the C-SOADAS is the addition of the evaluation of dosing regimen convenience, which was actually included in the original draft version of the SOADAS but was excluded from the final version. We decided to add this question because of experts' suggestion at the development phase. ${ }^{11}$ The analysis results showed that the convenience item was highly correlated with total score, and no respondent had difficulty understanding or answering this question. However, a concern was raised from our observations with regard to the item examining an OAD's effect on weight. A number of participants had difficulty answering this question because they tended to attribute the change in weight to their diet rather than the drug's effect. Further examination of the appropriateness and psychometric properties of this item is needed.

For a chronic condition such as DM, it is important to take into account a patient's perception of treatment outcomes. Indeed, it has been found that improvements in patient treatment satisfaction can improve treatment efficacy and adherence. ${ }^{19}$ The validated C-SOADAS could be used as an outcome measure in the future clinical trials of OADs that involve Chinese DM patients. It could also be incorporated into routine clinical practice as a quick assessment of OAD treatment from a patient's perspective and as a way of identifying patients' concerns or problems with their treatment. To enhance C-SOADAS' usefulness and applicability, future study is needed to determine its minimal clinically important difference.

There are several limitations to this study. First, the study results may not be able to generalize to all T2DM patients due to the fact that our study participants were a convenience sample recruited from the outpatient clinics at a single hospital in Taiwan. The study sample could be representative of relatively healthy DM patients. Second, the responsiveness of the C-SOADAS could not be assessed due to the cross-sectional design of the current study. Third, although efforts were made to encourage the participants to give honest answers, ensuring them that their replies would have no consequences, socially desirable responses may not have been fully avoided, particularly to the C-SOADAS questions. 


\section{Conclusion}

The 5-item C-SOADAS appears to be a psychometrically acceptable measure of OAD treatment satisfaction among T2DM patients in Taiwan. The C-SOADAS could be incorporated into clinical practice as a quick and useful tool that provides health care professionals with a good understanding of OAD treatment outcomes from patients' perspective. Future study is needed to determine C-SOADAS' minimal clinically important differences and to assess its responsiveness to detect change over time.

\section{Keypoints}

1. The Chinese version of the Satisfaction with Oral AntiDiabetic Agent Scale (C-SOADAS) is a psychometrically acceptable measure of oral antidiabetic drug (OAD) treatment satisfaction among type 2 diabetes mellitus (DM) patients in Taiwan.

2. The developed and validated C-SOADAS could be incorporated into clinical practice as a quick and useful tool that provides health care professionals with a good understanding of OAD treatment outcomes from patients' perspective.

\section{Acknowledgment}

This study was sponsored by AstraZeneca Taiwan Ltd (A-104-062) and the Shin Kong Ho-Su Memorial Hospital (SKH-8302-105-NDR04).

\section{Disclosure}

The authors report no conflicts of interest in this work.

\section{References}

1. International Diabetes Federation [webpage on the Internet]. IDF Western Pacific Members. Available from: https://www.idf.org/our-network/ regions-members/western-pacific/members/114-taiwan.html. Accessed March 28, 2018.

2. Chan JCN, Malik V, Kadowaki T, et al. Diabetes in Asia: epidemiology, risk factors, and pathophysiology. JAMA. 2009;301(20):2129-2140.

3. World Health Organization. Global Report on Diabetes. Geneva: World Health Organization; 2016
4. Prevalence of Diabetes Mellitus [webpage on the Internet]. Available from: http://data.gov.tw/node/9337. Accessed August 14, 2017.

5. Bullens PH, van Loon CJ, de Waal Malefijt MC, Laan RF, Veth RP. Patient satisfaction after total knee arthroplasty: a comparison between subjective and objective outcome assessments. J Arthroplasty. 2001; 16(6):740-747.

6. El Achhab Y, Nejjari C, Chikri M, Lyoussi B. Disease-specific healthrelated quality of life instruments among adults diabetic: a systematic review. Diabetes Res Clin Pract. 2008;80(2):171-184.

7. Levterova BA, Dimitrova DD, Levterov GE, Dragova EA. Instruments for disease-specific quality-of-life measurement in patients with type 2 diabetes mellitus - a systematic review. Folia Med. 2013; 55(1):83-92.

8. Shikiar R, Rentz AM. Satisfaction with medication: an overview of conceptual, methodologic, and regulatory issues. Value Health. 2004; 7(2):204-215

9. Centers for Disease Control and Prevention [webpage on the Internet]. Age-Adjusted Percentage of Adults with Diabetes Using Diabetes Medication, by Type of Medication, United States, 1997-2011. Available from: https://www.cdc.gov/diabetes/statistics/meduse/fig2.htm. Accessed August 31, 2017.

10. Chang TJ, Jiang YD, Chang $\mathrm{CH}$, Chung $\mathrm{CH}$, Yu NC, Chuang LM. Accountability, utilization and providers for diabetes management in Taiwan, 2000-2009: an analysis of the National Health Insurance database. J Formos Med Assoc. 2012;111(11):605-616.

11. Donatti C, Wild D, Horblyuk R, et al. Psychometric evaluation of the Satisfaction with Oral Anti-Diabetic Agent Scale (SOADAS). Diabetes Res Clin Pract. 2008;80(1):108-113.

12. EuroQol Office [webpage on the Internet]. EQ-5D About. Available from: https://euroqol.org/eq-5d-instruments/eq-5d-31-about/. Accessed August 23, 2017.

13. Tsuchiya A, Ikeda S, Ikegami N, et al. Estimating an EQ-5D population value set: the case of Japan. Health Econ. 2002;11(4):341-353.

14. Nunnally J, Bernstein I. Psychometric Theory. New York: McGraw-Hill Higher, INC; 1994

15. Kontodimopoulos N, Arvanitaki E, Aletras VH, Niakas D. Psychometric properties of the Greek Diabetes Treatment Satisfaction Questionnaire. Health Qual Life Outcomes. 2012;10:17.

16. RedekopWK, KoopmanschapMA, StolkRP, Rutten GE, WolffenbuttelBH, Niessen LW. Health-related quality of life and treatment satisfaction in Dutch patients with type 2 diabetes. Diabetes Care. 2002;25(3): 458-463.

17. American Diabetes Association [webpage on the Internet]. Diabetes Symptoms. Available from: http://www.diabetes.org/diabetesbasics/symptoms/?referrer=https://www.google.com.tw/. Accessed September 8, 2017.

18. Li HY, Jiang YD, Chang CH, Chung CH, Lin BJ, Chuang LM. Mortality trends in patients with diabetes in Taiwan: a nationwide survey in 2000-2009. J Formos Med Assoc. 2012;111(11):645-650.

19. Brod M, Cobden D, Lammert M, Bushnell D, Raskin P. Examining correlates of treatment satisfaction for injectable insulin in type 2 diabetes: lessons learned from a clinical trial comparing biphasic and basal analogues. Health Qual Life Outcomes. 2007;5:8.
Patient Preference and Adherence

\section{Publish your work in this journal}

Patient Preference and Adherence is an international, peer-reviewed, open access journal that focuses on the growing importance of patient preference and adherence throughout the therapeutic continuum. Patient satisfaction, acceptability, quality of life, compliance, persistence and their role in developing new therapeutic modalities and compounds to optimize

\section{Dovepress}

clinical outcomes for existing disease states are major areas of interest for the journal. This journal has been accepted for indexing on PubMed Central The manuscript management system is completely online and includes a very quick and fair peer-review system, which is all easy to use. Visit http://www. dovepress.com/testimonials.php to read real quotes from published authors. 